Received: 13 December 2016 Accepted: 30 May 2017 Published online: 21 July 2017

\section{Unconventional band inversion and intrinsic quantum spin Hall effect in functionalized group-V binary films}

\author{
Sheng-shi Li ${ }^{1}$, Wei-xiao $\mathrm{Ji}^{2}$, Ping Li ${ }^{2}$, Shu-jun $\mathrm{Hu}^{1}$, Tie Zhou ${ }^{1}$, Chang-wen Zhang ${ }^{2}$ \& \\ Shi-shen Yan (iD ${ }^{1}$
}

Adequately understanding band inversion mechanism, one of the significant representations of topological phase, has substantial implications for design and regulation of topological insulators (TIs). Here, by identifying an unconventional band inversion, we propose an intrinsic quantum spin Hall (QSH) effect in iodinated group-V binary $\left(\mathrm{ABI}_{2}\right)$ monolayers with a bulk gap as large as $0.409 \mathrm{eV}$, guaranteeing its viable application at room temperature. The nontrivial topological characters, which can be established by explicit demonstration of $Z_{2}$ invariant and gapless helical edge states, are derived from the band inversion of antibonding states of $p_{x, y}$ orbitals at the $K$ point. Furthermore, the topological properties are tunable under strain engineering and external electric field, which supplies a route to manipulate the spin/charge conductance of edge states. These findings not only provide a new platform to better understand the underlying origin of QSH effect in functionalized group-V films, but also are highly desirable to design large-gap QSH insulators for practical applications in spintronics.

Topological insulators (TIs) ${ }^{1-3}$, a new quantum state of matter, which can effectively achieve the objective of spintronics by controlling spin configuration and spin current ${ }^{4,5}$, have attracted great research enthusiasm since they were proposed in 2005. A fascinating hallmark of TIs is the existence of time-reversal-protected edge states or surface states which span insulating bulk state and show a robustness against nonmagnetic perturbations. In view of these advantages, TIs are quite promising for application in spintronics ${ }^{6}$ and quantum computations ${ }^{7,8}$. To date, many three-dimensional (3D) materials have confirmed experimentally to be TIs, such as $\mathrm{Bi}_{2} \mathrm{Se}_{3}{ }^{9}$. However, tuning the Fermi level of 3D TIs still faces challenge, and the bulk carriers commonly exceed the surface carriers. By contrast, two-dimensional (2D) TIs, also known as quantum spin Hall (QSH) insulators, are more advantageous over 3D TIs since the electrons can only propagate along two directions with opposite spin and thus backscattering caused by nonmagnetic defects is completely forbidden, while the surface states of 3D TI are only free from exact $180^{\circ}$ backscattering. Besides, from the perspective of practical application, $2 \mathrm{D}$ TIs can easily actualize functionality and integration of devices due to their lower dimensionality. Nevertheless, the experimental process with regard to $2 \mathrm{D}$ TIs confines to $\mathrm{HgTe} / \mathrm{CdTe}^{7,8}$ and InAs/ $\mathrm{GaSb}^{10}$ quantum wells. The QSH effect of these quantum wells can only be observed under a harsh condition because of their small bulk gap, which severely blocks their application at room temperature. Hence, searching for novel 2D TIs which possess experimental feasibility and admirable QSH effect with large bulk gap is desirable.

Following the first proposed QSH insulator of graphene ${ }^{11}$, other 2D group-IV films, such as silicene ${ }^{12}$, germanene ${ }^{12}$, stanene $\mathrm{e}^{13,14}$ and plumbene ${ }^{15}$, are verified as 2D TIs successively. These hexagonal honeycomb structures provide a certain reference on searching for QSH insulator. For example, the graphene-like structures comprised of group-V element trigger considerable research attention, including $\mathrm{Bi}(111)$ bilayer (bismuthene) ${ }^{16}$, antimonene ${ }^{17}$ and arsenen $e^{18}$ films. Among them, the bismuthene is predicted as an intrinsic QSH insulator due to its stronger spin-orbit coupling (SOC) strength, and it has been successfully prepared on $\mathrm{Bi}_{2} \mathrm{Te}_{3}$ and $\mathrm{Bi}(111$ ) substrates ${ }^{16,19,20}$. For atimonene and arsenene, which are normal insulators (NIs) in ground state, they can transform into 2D TIs with assistance of appropriate strain engineering ${ }^{21-23}$, accompanied with band inversion ${ }^{24}$ of $p_{\mathrm{x}, \mathrm{y}}-p_{\mathrm{z}}$ at $\Gamma$ point. Interestingly, similar results are also demonstrated in group- $\mathrm{V}$ binary monolayers, but they are

${ }^{1}$ School of Physics, State Key Laboratory of Crystal Materials, Shandong University, Jinan, Shandong, 250100, P.R. China. ${ }^{2}$ School of Physics and Technology, University of Jinan, Jinan, Shandong, 250022, P.R. China. Correspondence and requests for materials should be addressed to S.-s.Y. (email: shishenyan@sdu.edu.cn) C.-w.Z. (email: ss_zhangchw@ujn.edu.cn) 
thermodynamically instable with softened modes and imaginary frequency ${ }^{25}$. In fact, there also has possibility to prepare group- $\mathrm{V}$ binary monolayers, such as $\mathrm{SbAs}^{26}$, whose bulk material is naturally existing.

In experiments, the realization of strain engineering still faces challenges, especially for the operating range exceeding $10 \%$. Chemical functionalization, as an alternative and useful route to introduce intrinsic topological phase, is proposed ${ }^{14,15,27-34}$. Such manipulation not only facilitates the regulation of electronic and topological properties, but also protects the desirable properties from environmental degradation. Recently, intensive efforts have implemented on functionalized group-V films ${ }^{31,33,35-40}$ which can readily achieve intrinsic nontrivial topology and shows classic Kane-Mele-type QSH effect. However, the topological mechanism of these systems is still a mystery topic to be further explored and understood. In view of above-mentioned group-V binary monolayers, two questions spontaneously arise: (i) can chemical functionalization simultaneously improve the stability and realize intrinsic QSH effect in group-V binary monolayers? (ii) If so, what is the potential topological mechanism, namely, how to characterize this topological nature?

In this work, based on first-principles calculations, we propose the simultaneous presence of unconventional band inversion and intrinsic QSH effect in iodinated group-V binary monolayers $\left(\mathrm{ABI}_{2}\right)$ which are dynamically and thermally stable at room temperature. The topological phase, accompanied with a sufficiently large bulk gap of $0.409 \mathrm{eV}$, can be identified by the band inversion of antibonding states of $p_{\mathrm{x}, \mathrm{y}}$ orbitals between $\mathrm{A}$ and $\mathrm{B}$ atoms induced by SOC at $\mathrm{K}$ point and explicit confirmation of $\mathrm{Z}_{2}$ invariant, as well as gapless helical edge states. In addition, their QSH effect can be modulated by strain engineering and external electric field with tunable bulk gap. The electronically controlled transition between nontrivial and trivial phases in $\mathrm{AsBiI}_{2}$ monolayer can effectively switch spin and charge transports to design topological quantum devices. These findings are conducive to propelling the understanding of topological mechanism in group- $\mathrm{V}$ films and supporting promising candidates to design spintronic and optoelectronic devices.

\section{Computational Details}

All first-principles calculations based on density functional theory (DFT) are carried out using Vienna Ab-Initio Simulation Package (VASP) ${ }^{41}$, 42 . The projector-augmented wave (PAW) method $^{43}$ is used to describe the electron-ion potential, and the Generalized gradient approximation (GGA) in Perdew-Burke-Ernzerhof (PBE) ${ }^{44,45}$ form is adapted to approximate the electron-electron interaction. The SOC interaction is included in the step of self-consistent calculations of electronic structure. The kinetic energy cutoff of plane wave basis is set as $500 \mathrm{eV}$. A unit cell with periodic boundary condition is employed, and a vacuum space of $20 \AA$ is applied. We employ the k-meshes of $11 \times 11 \times 1$ and $15 \times 15 \times 1$ for geometry optimization and self-consistent electronic structure calculations, respectively. All lattice constants and atom coordinates are optimized with the self-consistent criteria of $10^{-6} \mathrm{eV}$ until the convergence of force on each atom is less than $0.001 \mathrm{eV} / \AA$. Hybrid HSE06 functional ${ }^{46}$ is used to confirm the band structure of functionalized systems. The phonon spectra are calculated using a supercell approach within the PHONOPY code ${ }^{47}$.

The calculations of edge states for $\mathrm{ABI}_{2}$ monolayers are implemented by Wannier 90 package ${ }^{48}$. Based on maximally localized Wannier functions (MLWFs), an iterative Green's function method ${ }^{49,50}$ is employed to calculate the edge states of a semi-infinite lattice and utilized to observer the local density of state (LDOS) of the edges.

\section{Results}

The group- $\mathrm{V}$ binary monolayers, comprised of two elements from $\mathrm{P}$ to $\mathrm{Bi}$, contain a total of six situations (PAs, $\mathrm{PSb}, \mathrm{PBi}, \mathrm{AsSb}, \mathrm{AsBi}, \mathrm{SbBi}$ ). Here, they are collectively represented as AB monolayers, where $\mathrm{A}$ and $\mathrm{B}$ atoms are defined as the elements with minor and greater atomic number, respectively. Figure 1(a) depicts the side view of pristine $\mathrm{AB}$ monolayers, which obtains a buckled configuration. The $\mathrm{AB}$ monolayers are reported kinetically stable except that AsBi and SbBi monolayers possess softened modes and imaginary frequency near $\Gamma$ point ${ }^{25}$. According to previously theoretical works ${ }^{29,51}$, the QSH effect is strongly associated with the SOC strength of functional atoms, thus we choose to passivate the monolayers using iodine atom to maximize the SOC interaction. Here, the iodinated monolayers are denoted as $\mathrm{ABI}_{2}\left(\mathrm{PAsI}_{2}, \mathrm{PSbI}_{2}, \mathrm{PBiI}_{2}, \mathrm{AsSbI}_{2}, \mathrm{AsBiI}_{2}, \mathrm{SbBiI}_{2}\right)$, and their geometric structures are presented in Fig. 1(b,c), along with corresponding 2D Brillouin zone in Fig. 1(d). Compared with pristine cases, the $\mathrm{ABI}_{2}$ monolayers prefer a quasi-planar configuration with iodine atoms alternately decorated on both sides of AB monolayers (space group P3M1, No. 156). Actually, the case of iodination on one side is also investigated, the fact demonstrates the functionalized monolayers are unstable after structural optimization, which may be attributed to the coulomb repulsion between iodine atoms and its smaller electronegativity. So we primarily concentrate on the configuration with decoration on both sides. In Fig. 1(e), we present the lattice constants of pristine and functionalized $\mathrm{AB}$ monolayers. The former is in good agreement with previous results ${ }^{25}$, while the latter indicates that $\mathrm{AB}$ monolayers are stretched under the effect of iodination, accompanied with buckled height $(h)$ reducing to around $0.1 \AA$, as illustrated in the insert of Fig. 1(e). From Table 1 , one can also see that, as the rise of atomic number of component atoms, both lattice constant and A-B bond length increase gradually in order of $\mathrm{PAsI}_{2}<\mathrm{PSbI}_{2}<\mathrm{PBiI}_{2}<\mathrm{AsSbI}_{2}<\mathrm{AsBiI}_{2}<\mathrm{SbBiI}_{2}$.

The thermodynamic stability of $\mathrm{ABI}_{2}$ monolayers is evaluated by the formation energy which is defined as:

$$
E_{f}=E\left(\mathrm{ABI}_{2}\right)-n_{\mathrm{A}} E_{\mathrm{A}}-n_{\mathrm{B}} E_{\mathrm{B}}-n_{\mathrm{I}} E_{\mathrm{I}},
$$

where $E\left(\mathrm{ABI}_{2}\right)$ represents the total energies of iodinated $\mathrm{AB}$ monolayers. $E_{\mathrm{A}}, E_{\mathrm{B}}$ and $E_{\mathrm{I}}$ are the chemical potential of $\mathrm{A}, \mathrm{B}$ and iodine atoms obtained from bulk bismuth, bulk antimony, bulk arsenic, black phosphorus and bulk iodine, respectively. The $n_{\mathrm{A}}, n_{\mathrm{B}}$ and $n_{\mathrm{I}}$ are the numbers of $\mathrm{A}, \mathrm{B}$ and iodine atoms in the $\mathrm{ABI}_{2}$ unit cell. The calculated formation energies are listed in Table 1 . The negative values demonstrate that the iodination is an exothermal process, suggesting a higher thermodynamic stability. Moreover, the calculation of phonon spectrum is performed to check the kinetic stability of $\mathrm{ABI}_{2}$ monolayers, and we find that the iodination can effectively 
(a)

$\mathrm{B}$

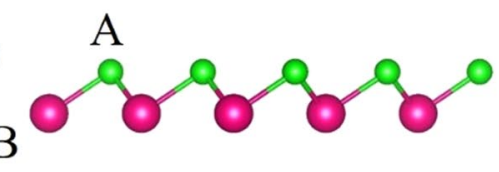

(b)

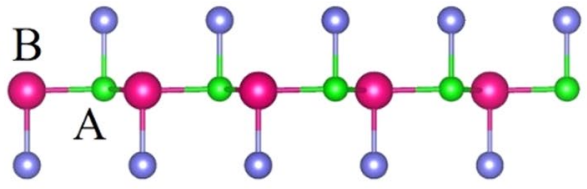

(e)

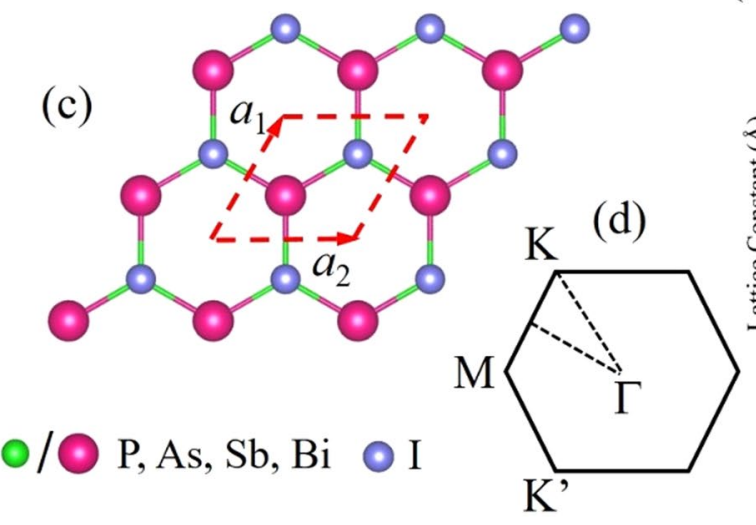

Figure 1. (a) Atomic structure of pristine $\mathrm{AB}$ monolayers. (b) Side view and (c) top view of $\mathrm{ABI}_{2}$ monolayer. (d) The corresponding $2 \mathrm{D}$ Brillouin zone of $\mathrm{ABI}_{2}$ monolayer with high-symmetry points. (e) Evolution of lattice constant for pristine $\mathrm{AB}, \mathrm{ABH}_{2}, \mathrm{ABI}_{2}$ monolayers, respectively. The insert stands for the buckled height $(h)$ as a function of different configurations.

\begin{tabular}{|l|l|l|l|l|l|l|l|l|}
\hline Configurations & $\boldsymbol{a}(\AA)$ & $\boldsymbol{d}_{\mathrm{A}-\mathbf{B}}(\AA)$ & $\boldsymbol{h}(\AA)$ & $\boldsymbol{E}_{f}(\mathbf{e V} /$ unit cell $)$ & $\boldsymbol{E}_{\mathbf{k}}(\mathbf{e V})$ & $\boldsymbol{E}_{\mathrm{g}}(\mathbf{e V})$ & $\boldsymbol{E}_{\boldsymbol{g}}^{\text {SOC }}(\mathbf{e V})$ & Property \\
\hline $\mathrm{PAsI}_{2}$ & 4.517 & 2.613 & 0.161 & -1.901 & 0.443 & 0.443 & 0.147 & NI \\
\hline $\mathrm{PSbI}_{2}$ & 4.807 & 2.777 & 0.101 & -2.452 & 0.828 & 0.788 & 0.567 & NI \\
\hline $\mathrm{PBiI}_{2}$ & 4.935 & 2.853 & 0.133 & -2.437 & 1.258 & 1.225 & 0.320 & NI \\
\hline $\mathrm{AsSb}_{2}$ & 4.983 & 2.877 & 0.064 & -3.287 & 0.402 & 0.402 & 0.082 & NI \\
\hline AsBiI $_{2}$ & 5.082 & 2.935 & 0.067 & -3.267 & 0.831 & 0.831 & 0.067 & TI \\
\hline $\mathrm{SbBiI}_{2}$ & 5.389 & 3.112 & 0.024 & -3.669 & 0.380 & 0.380 & 0.409 & TI \\
\hline
\end{tabular}

Table 1. Calculated lattice constant $(a)$, bond length $\left(d_{\mathrm{A}-\mathrm{B}}\right)$, buckled height $(h)$, formation energy $\left(E_{f}\right)$ and material property of $\mathrm{ABI}_{2}$ monolayers. $E_{\mathrm{k}}$ and $E_{\mathrm{g}}$ represent the band gap opening at $\mathrm{K}$ point and global band gap excluding SOC, respectively. $E_{g}^{S O C}$ denotes the SOC-induced band gap.

remove the softened modes and imaginary frequency in AsBi and SbBi monolayers, as shown in Fig. 2(a) and Fig. S1 in Supplementary Information. Similar results are also established for the other configurations, as depicted in Fig. S1.

On the other hand, we perform ab initio molecular dynamics (MD) simulations using a $3 \times 3 \times 1$ supercell at various temperature with a time step of $2 \mathrm{fs}$ to explore the structural perturbation of $\mathrm{ABI}_{2}$ monolayers during the 3 ps simulation. Taking $\mathrm{AsBiI}_{2}$ monolayer as example, the honeycomb lattice deforms slightly at $300 \mathrm{~K}$ in Fig. 2 (b). As the temperature increases to $400 \mathrm{~K}$, the geometric structure suffers a serious distortion, but no bond breaking can be observed, as illustrated in Fig. 2(c), indicating that the $\mathrm{AsBiI}_{2}$ monolayer could be steadily survived at room temperature. The thermal stabilities for other $\mathrm{ABI}_{2}$ systems are also displayed in Fig. S1. As a consequence, all $\mathrm{ABI}_{2}$ monolayers possess favorable stability, which is very significant for practical applications.

To reveal the prominent influence of iodination on the electronic properties of AB monolayers, we firstly present the calculated band structures of pristine AB monolayers in Fig. S2. Regardless of SOC, they exhibit semiconducting properties, while the introduction of SOC just modifies the size of band gap due to band splitting. As a result, the $\mathrm{QSH}$ effect is absent for $\mathrm{AB}$ monolayers, indicating a trivial topological phase. Through projecting the energy bands with atomic orbitals, one can see that the states near the Fermi level at the $\Gamma$ point mainly derive from the $p_{\mathrm{z}}$ and $p_{\mathrm{x}, \mathrm{y}}$ orbitals.

Next, we turn to the electronic properties of $\mathrm{ABI}_{2}$ monolayers. Here, the $\mathrm{PAsI}_{2}$ and $\mathrm{SbBiI}_{2}$ are chosen as the representatives and their band structures are presented in Fig. 3. For $\mathrm{PAsI}_{2}$ monolayer, it shows a semiconducting property with a direct band gap $\left(E_{\mathrm{K}}\right)$ of $0.443 \mathrm{eV}$ located at the $\mathrm{K}$ point when excluding SOC, which is distinctly different from the semi-metallicity in functionalized $\mathrm{Bi}(111)$ bilayer $^{31,38-40,52}$, as illustrated in Fig. 3(a). Similar band features can be obtained in other configurations, except for $\mathrm{PSbI}_{2}$ and $\mathrm{PBiI}_{2}$ monolayers which show indirect global band gaps $\left(E_{\mathrm{g}}\right)$ due to the deviation of conduction bands minimum (CBM), see Fig. S3. As the SOC is taken into account, the most conspicuous change is the removal of degeneracy of energy bands owing to the lack of 


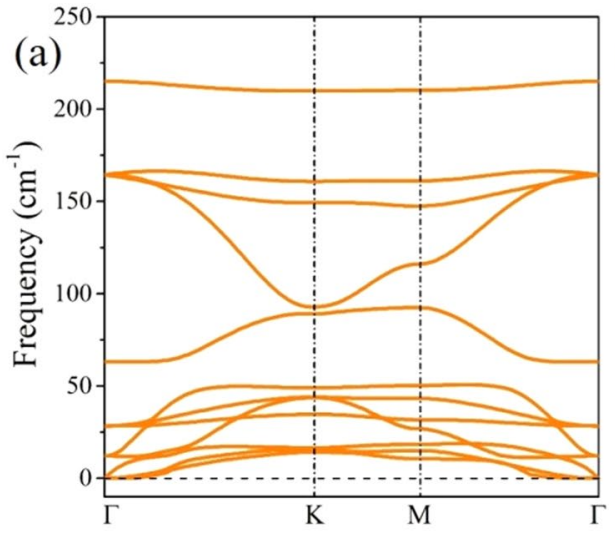

(b)

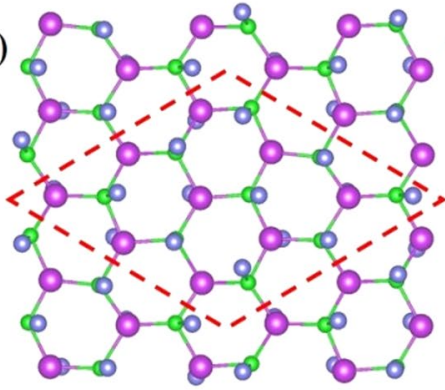

$300 \mathrm{~K}$ (c)

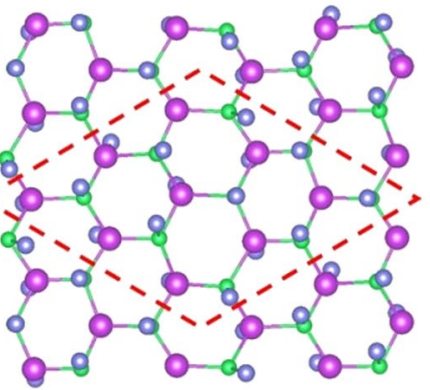

$400 \mathrm{~K}$

Figure 2. (a) Calculated phonon spectrum of $\mathrm{AsBiI}_{2}$ monolayer. Corresponding molecular dynamics (MD) simulation for a $3 \times 3$ supercell at $300 \mathrm{~K}(\mathbf{b})$ and $400 \mathrm{~K}(\mathbf{c})$ after 3 ps, respectively.
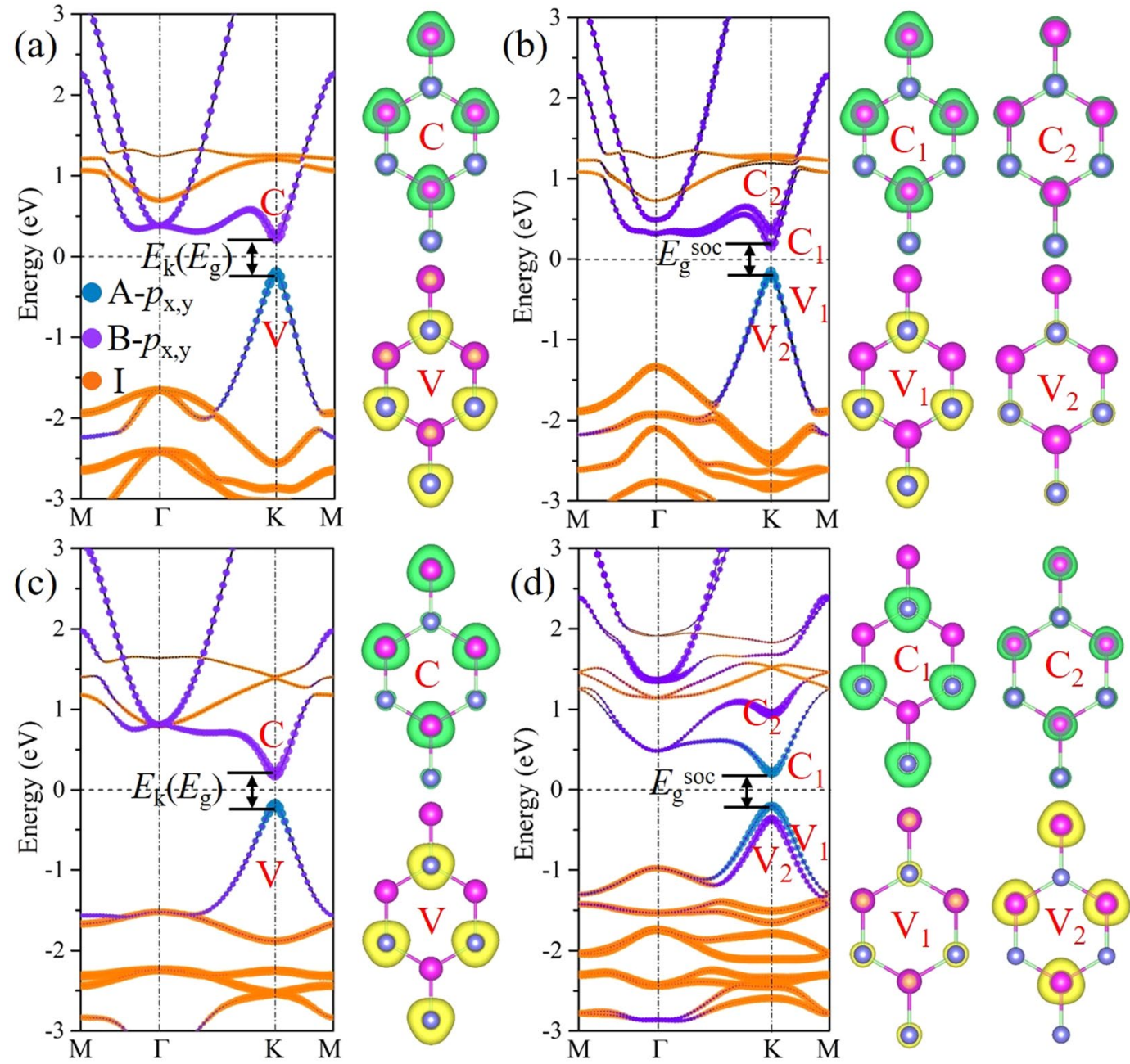

Figure 3. Orbital-resolved band structures and partial charge density of VBM and CBM. (a) $\mathrm{PAsI}_{2}$ monolayer without SOC, (b) $\mathrm{PAsI}_{2}$ monolayer with SOC, (c) $\mathrm{SbBiI}_{2}$ monolayer without SOC and (d) $\mathrm{SbBiI}_{2}$ monolayers with SOC.

spatial inversion symmetry. We find that the valence bands maximum (VBM) and $\mathrm{CBM}$ of $\mathrm{ABI}_{2}$ monolayers are all fixed at K point, forming a direct band gap $\left(E_{g}^{S O C}\right)$. Compared with the cases without SOC, the band gaps of $\mathrm{ABI}_{2}$ monolayers are decreased except for $\mathrm{SbBiI}_{2}$ monolayer, as listed in Table 1. 
(a)

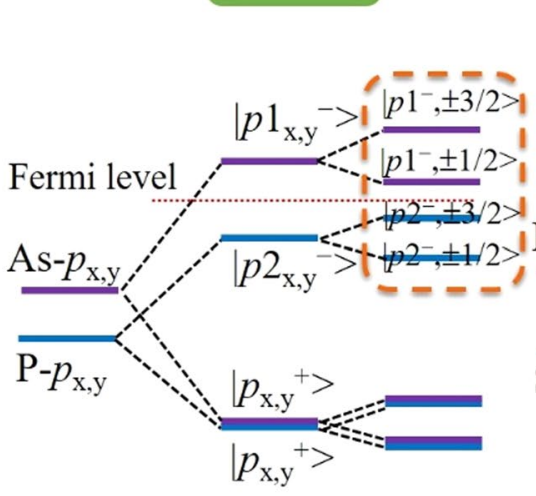

(I)
(II) (b)

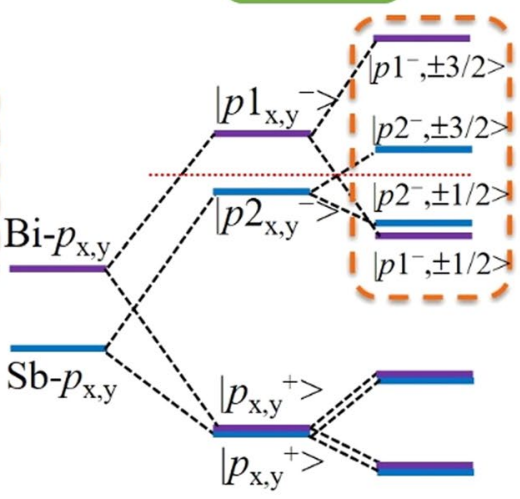

(I)
(II) (c)

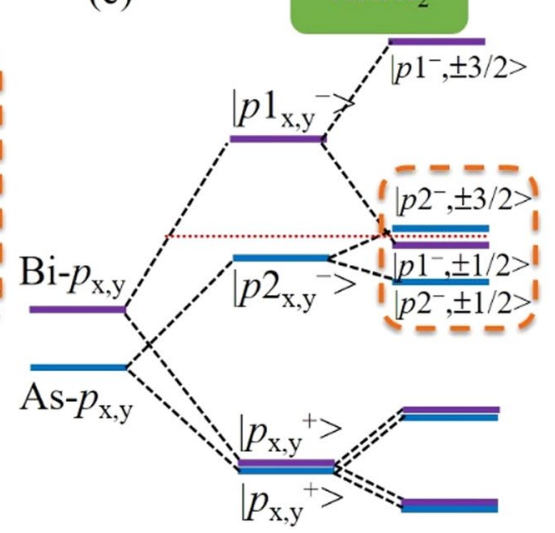

(I)

(II)

Figure 4. The evolution of $p_{\mathrm{x}, \mathrm{y}}$ orbitals of $\mathrm{A}$ and $\mathrm{B}$ atoms at $\mathrm{K}$ point near the Fermi level under chemical bonding (I) and SOC (II) effects. (a) $\mathrm{PAsI}_{2}$, (b) $\mathrm{SbBiI}_{2}$, and (c) $\mathrm{AsBiI}_{2}$ monolayers.

In order to get a deeper insight into the band structure feature, we project energy bands onto different atomic orbitals. Regardless of SOC, for $\mathrm{PAsI}_{2}$ monolayer, the $\mathrm{P}-\mathrm{p}_{\mathrm{x}, \mathrm{y}}$ and As- $\mathrm{p}_{\mathrm{x}, \mathrm{y}}$ orbitals are distinctly separated in the vicinity of Fermi level and contribute to the VBM and CBM, respectively. The partial charge densities with respect to VBM and CBM are further calculated. One can see that the VBM and CBM are localized at the P and As sites, respectively, as shown in Fig. 3(a). When considering SOC effect, the contributions of atomic orbitals at VBM and CBM are not altered, as evidenced in Fig. 3(b). Similar to $\mathrm{PAsI}_{2}$ monolayer, the VBM and CBM of $\mathrm{SbBiI}_{2}$ monolayer are derived from $S b-p_{x, y}$ and $B i-p_{x, y}$ orbitals without SOC, as shown in Fig. 3(c). However, the stronger SOC effect triggers the realignment of band order. Here, both VBM and CBM are mainly originated from the $\mathrm{Sb}-p_{\mathrm{x}, \mathrm{y}}$ orbitals, whereas the energy band contributed by Bi- $p_{\mathrm{x}, \mathrm{y}}$ orbitals reverses into valance band, being the second highest occupied state, see Fig. 3(d). The partial charge densities of these four bands at K point are in good agreement with the orbital-resolved band structure. Such SOC induced band inversion between Sb- $p_{\mathrm{x}, \mathrm{y}}$ and Bi- $p_{\mathrm{x}, \mathrm{y}}$ strongly points to the presence of QSH effect. Similar results can be observed in $\mathrm{AsBiI}_{2}$ monolayer, while the other monolayers are not provided with this form of band inversion, as shown in Fig. S3.

To understand the physical mechanism underlying the band inversion, we turn to the effects of chemical bonding and $\mathrm{SOC}$ on energy bands at $\mathrm{K}$ point for $\mathrm{ABI}_{2}$ monolayers and the schematic diagrams are illustrated in Fig. 4. Here, only the evolution of $p_{\mathrm{x}, \mathrm{y}}$ orbitals of A and B atoms is focused on near the Fermi level, since the $p_{z}$ orbitals are saturated by iodine atoms. Starting from $\mathrm{PAsI}_{2}$ monolayer, in stage (I), the formation of chemical bonding makes the $p_{\mathrm{x}, \mathrm{y}}$ orbitals split into bonding and antibonding states, i. e., $\left|p_{\mathrm{x}, \mathrm{y}}^{+}\right\rangle$and $\left|p_{\mathrm{x}, \mathrm{y}}^{-}\right\rangle$, where the superscripts + and - represent the bonding and antibonding states. For convenience, the states near the Fermi level are labeled as $\left|p 1_{\mathrm{x}, \mathrm{y}}^{-}\right\rangle$and $\left|p 2_{\mathrm{x}, \mathrm{y}}^{-}\right\rangle$, as illustrated in Fig. 4(a), which are respectively originated from As and $\mathrm{P}$ atoms. The $\left|p 1_{\mathrm{x}, \mathrm{y}}^{-}\right\rangle$locates above $\left|p 2_{\mathrm{x}, \mathrm{y}}^{-}\right\rangle$, yielding a sizeable band gap. When SOC is turned on in stage (II), the degeneracy of the $\left|p_{\mathrm{x}, \mathrm{y}}^{-}\right\rangle$is lifted, resulting in the decrease of the band gap, but the sequence of energy level is unchanged, as shown in Fig. 4(a). Similar situation is obtained in $\mathrm{PSbI}_{2}, \mathrm{PBiI}_{2}$ and $\mathrm{AsSbI}_{2}$ monolayers. For $\mathrm{SbBiI}_{2}$ monolayer, a greater orbital splitting is generated due to its larger strength of $\mathrm{SOC}^{53}$, driving $\left|p 1^{-}, \pm 1 / 2\right\rangle$ directly downshift below $\left|p 2^{-}, \pm 1 / 2\right\rangle$ and improving $\left|p 2^{-}, \pm 3 / 2\right\rangle$ above the Fermi level, thus introduces a significant band inversion, see Fig. 4(b). Meanwhile, we find that the $\mathrm{AsBiI}_{2}$ monolayer also possesses band inversion, in which the $\mid p 1^{-}$, $\pm 1 / 2\rangle$ and $\left|p 2^{-}, \pm 3 / 2\right\rangle$ are obviously reversed at the Fermi level, as shown in Fig. 4(c). In a word, the appearance of band inversion, to a great extent, is determined by the bonding and SOC strength of A and B atoms. It is important to notice that such band inversion associated with antibonding states of $p_{\mathrm{x}, \mathrm{y}}$ orbitals for $\mathrm{AsBiI}_{2}$ and $\mathrm{SbBiI}_{2}$ monolayers is distinctly different from the cases of functionalized III-V ${ }^{30}$ and IV films ${ }^{14,15,24,34,54}$, indicating that it is an unconventional mechanism to predict $2 \mathrm{D}$ TIs.

A QSH insulator is usually represented by a topological invariant $Z_{2}=1$. Thus, to verify our supposition, we calculate the $\mathrm{Z}_{2}$ invariant of $\mathrm{ABI}_{2}$ monolayers by the scheme proposed by Soluyanov and Vanderbilt ${ }^{55,56}$. This method tracks the evolution of Wannier Center of Charges (WCCs) for an effective 1D system with fixed $k_{\mathrm{y}}$ in the subspace of occupied bands. The Wannier functions (WF) in regard to lattice vector $R$ can be written as:

$$
|R, n\rangle=\frac{1}{2 \pi} \int_{-\pi}^{\pi} d k e^{-i k(R-x)}\left|u_{n k}\right\rangle .
$$

The WF depends on a gauge choice for the Bloch states $\left|u_{n k}\right\rangle$. A WCC $\bar{x}_{n}$ is defined as the mean value of $\langle 0 n|\hat{X}| 0 n\rangle$, where the $\hat{X}$ is the position operator and $|0 n\rangle$ is the state corresponding to a WF in the cell with $R=0$. Then we can obtain that: 

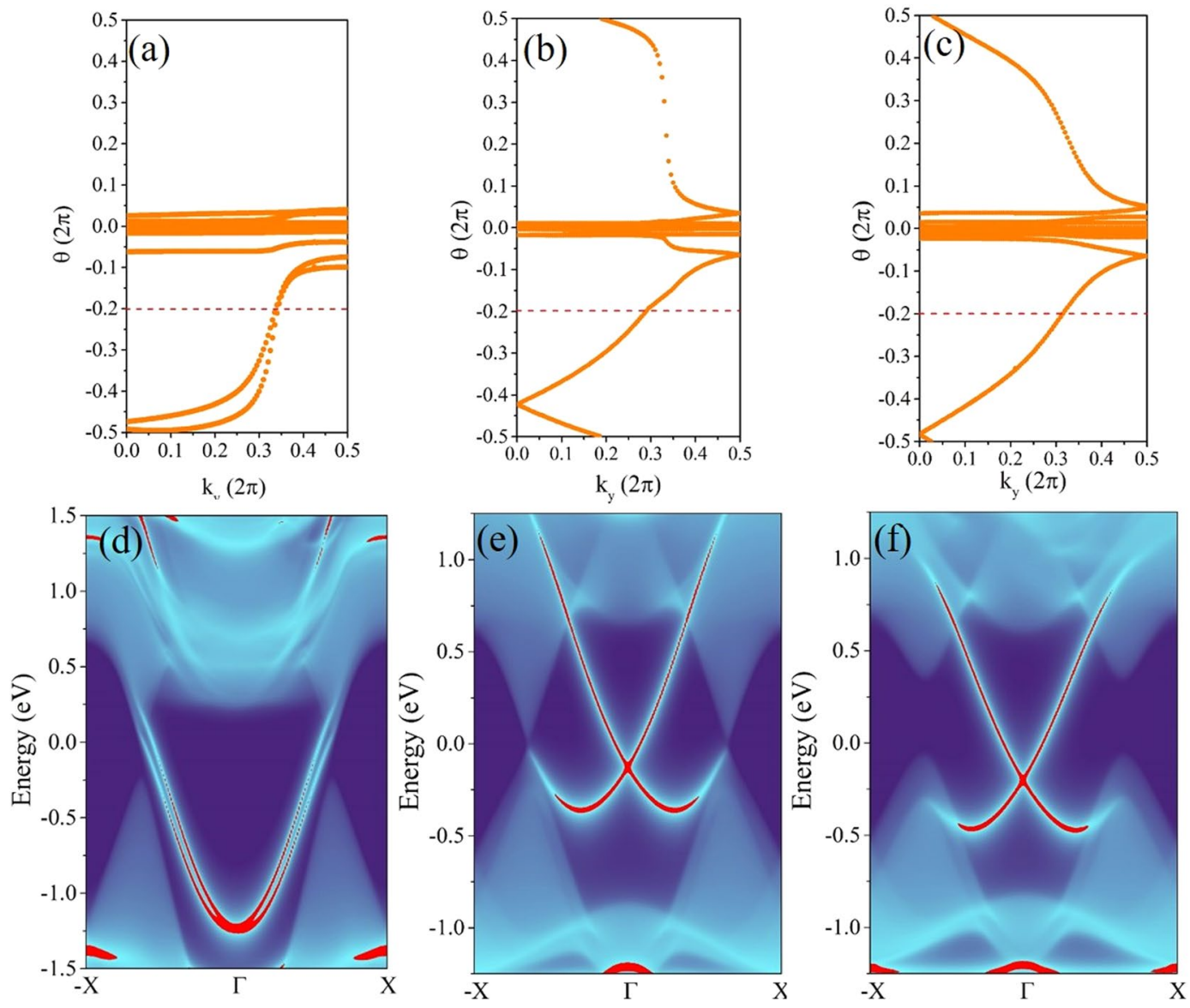

Figure 5. Evolution of the Wannier charge centers (WCCs) along $k_{\mathrm{y}}$ for (a) $\mathrm{PAsI}_{2}$, (b) $\mathrm{AsBiI}_{2}$ and (c) $\mathrm{SbBiI}_{2}$ monolayer. Calculated semi-infinite edge states of (d) $\mathrm{PAsI}_{2},(\mathbf{e}) \mathrm{AsBiI}_{2}$ and (f) $\mathrm{SbBiI}_{2}$ monolayers.

$$
\bar{x}_{n}=\frac{i}{2 \pi} \int_{-\pi}^{\pi} d k\left\langle u_{n k}\left|\partial_{k}\right| u_{n k}\right\rangle
$$

Assuming that $\sum_{\alpha} \bar{x}_{\alpha}^{S}=\frac{1}{2 \pi} \int_{B Z} A^{S}$ with $S=I$ or $I I$, where $\alpha$ is a band index of the occupied states and $I$ and $I I$ are the Kramer partners, while $A$ is the Berry connection. So the $\mathrm{Z}_{2}$ topological invariant can be expressed as:

$$
Z_{2}=\sum_{\alpha}\left[\bar{x}_{\alpha}^{I}\left(T R I M_{1}\right)-\bar{x}_{\alpha}^{I I}\left(T R I M_{1}\right)\right]-\sum_{\alpha}\left[\bar{x}_{\alpha}^{I}\left(T R I M_{2}\right)-\bar{x}_{\alpha}^{I I}\left(T R I M_{2}\right)\right]
$$

where TRIM represents the time-reversal-invariant momentum. The identification of $Z_{2}$ invariant can be obtained by counting the numbers of crossing between any arbitrary horizontal reference line and evolution of the WCCs, where the odd and even numbers represent nontrivial and trivial topological phase, respectively. Figure 5(a-c) present the evolution of the WCCs between two TRIM of the Brillouin zone for $\mathrm{PAsI}_{2}, \mathrm{AsBiI}_{2}$, and $\mathrm{SbBiI}_{2}$ monolayers. Corresponding to above analysis, both $\mathrm{AsBiI}_{2}$ and $\mathrm{SbBiI}_{2}$ monolayers are nontrivial QSH insulators with $\mathrm{Z}_{2}=1$. However, the PAsI $\mathrm{P}_{2}$ monolayer and the other configurations obtain a $\mathrm{Z}_{2}$ invariant of 0 , as depicted in Fig. S4, indicating a trivial topological phase.

The presence of topologically protected and spin-momentum locked edge states is one of the prominent features of QSH insulators. The LDOSs of edges for $\mathrm{ABI}_{2}$ monolayers are further calculated to confirm the topological properties exactly. For $\mathrm{PAsI}_{2}$ monolayer, as shown in Fig. 5(d), the edge states start from conduction band and then are absorbed back into the same conduction band rather than providing spin transport channels between valence and conduction bands, evidencing that $\mathrm{PAsI}_{2}$ monolayer is a trivial insulator. Similar LDOSs are verified in $\mathrm{PSbI}_{2}, \mathrm{PBiI}_{2}$, and $\mathrm{AsSbI}_{2}$ monolayers, see Fig. S5(a-c), indicating that they likewise belong to the scope of NIs. On the contrary, we notice that a pair of gapless edge states traverse across the bulk gap and connect the conduction and valence bands in $\mathrm{AsBiI}_{2}$ and $\mathrm{SbBiI}_{2}$ monolayers, as depicted in Fig. 5(e,f). By projecting the contribution of spin up and spin down component, see Fig. S5(d,e), we find that the spin-momenta of those states are locked, suggesting the counter-propagating edge states have opposite spin-polarization. Thus, we can readily ascertain the nontrivial topological phase in $\mathrm{AsBiI}_{2}$ and $\mathrm{SbBiI}_{2}$ monolayers. Moreover, apart from intrinsic QSH effect, the satisfactory bulk gaps guarantee their experimental observability and viable application at room temperature, especially for $\mathrm{SbBiI}_{2}$ monolayer, whose bulk gap attains a sufficiently large value of $0.409 \mathrm{eV}$. 


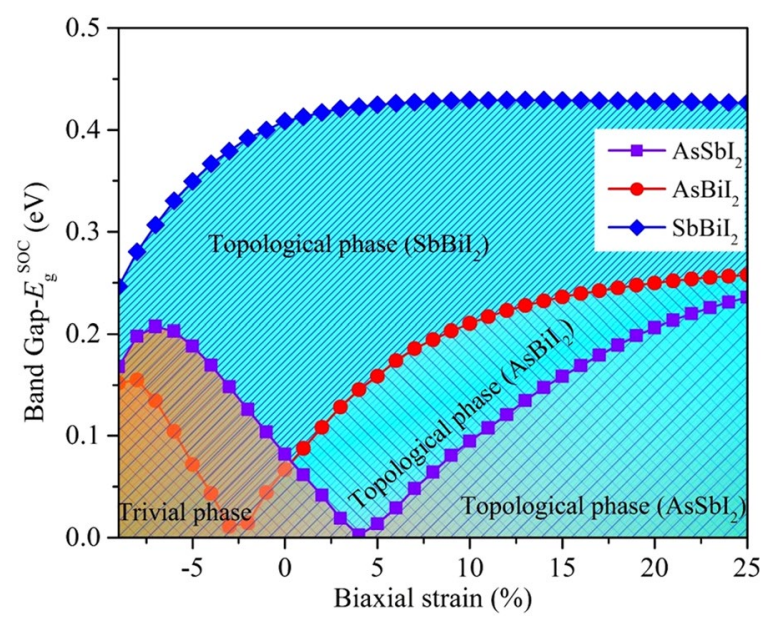

Figure 6. Variation of band gap $\left(E_{g}^{S O C}\right)$ as a function of biaxial strain for $\mathrm{AsSbI}_{2}, \mathrm{AsBiI}_{2}$, and $\mathrm{SbBiI}_{2}$ monolayers, respectively.

According to former analysis, under a fixed SOC strength, the band inversion also relates to the bond strength which can be tuned by strain engineering. To realize the transition of TI and enhance the bulk gaps, we employ in-plane biaxial strain on $\mathrm{ABI}_{2}$ monolayers, which equals changing the lattices as $\varepsilon=\left(a-a_{0}\right) / a_{0}$, where $a\left(a_{0}\right)$ is lattice constant under the strain (equilibrium) condition. Figure 6 presents the variation of band gap as a function of biaxial strain. One can see that the $\mathrm{SbBiI}_{2}$ monolayer maintains its topological nature in the range of $-9 \sim 20 \%$. Under tensile strain, the band gap increases monotonously and reaches a maximum of $0.429 \mathrm{eV}$ at $5 \%$. After that, it almost remains unchanged even if the tensile strain reaches $25 \%$. Instead, the compressive strain leads to the decrease of band gap. It can be interpreted by the fact that the shorter bond length drives the energy difference between $\left|p 2_{\mathrm{x}, \mathrm{y}}^{-}\right\rangle$and $\left|p 1_{\mathrm{x}, \mathrm{y}}^{-}\right\rangle$to reduce, and then the band inversion similar to $\mathrm{AsBiI}_{2}$ monolayer arises under SOC effect. To be brief, the SbBiI ${ }_{2}$ monolayer shows a favorable robustness of QSH effect against strain engineering. However, for $\mathrm{AsBiI}_{2}$ monolayer, the QSH effect can be easily tuned by strain engineering due to its relatively weak inversion strength. From Fig. 6, one can see that the band gap decreases firstly and then increases with the increasing compressive strain, in which the critical point of $-3 \%$ indicates the annihilation of band inversion between $\left|p 2^{-}, \pm 3 / 2\right\rangle$ and $\left|p 1^{-}, \pm 1 / 2\right\rangle$, resulting in a trivial topological phase. On the contrary, the tensile strain enlarges the band gap significantly, persisting the topological nature. Interestingly, the $\mathrm{AsSbI}_{2}$ monolayer, a NI at equilibrium state, can transform into QSH insulator under tensile strain at $4 \%$, and the continuously increasing strain would improves the band gap, demonstrating its potential application in spintronic devices. Furthermore, the tunability of the band gap and TI phase transition for $\mathrm{PAsI}_{2}, \mathrm{PSbI}_{2}$ and $\mathrm{PBiI}_{2}$ monolayers are also presented in Fig. S6. Owing to their rather weak SOC strength, the achievement of band inversion requires sufficiently small energy difference of $\left|p_{\mathrm{x}, \mathrm{y}}^{-}\right\rangle$between $\mathrm{A}$ and $\mathrm{B}$ atoms, which means that a prodigious tensile strain should be employed. The critical points for these configurations are $17 \%, 21 \%$ and $9 \%$, respectively, which are conducive to understand the physical mechanism rather than application in devices, because such large tensile strain still faces many challenges in experiments.

The facile mechanism of band inversion also provides a possibility to control the topological properties of $\mathrm{ABI}_{2}$ monolayers by external electric field. Taking $\mathrm{AsBiI}_{2}$ and $\mathrm{SbBiI}_{2}$ monolayers as representatives, Fig. $7(\mathrm{a}, \mathrm{b})$ present the variation of band gaps with respect to an external electric field perpendicular to the films. For AsBiI monolayer [Fig. 7(a)], the intrinsic QSH effect is persevered under the negative electric field, in which the band gap increases monotonically. Under positive electric field, the band gap drops rapidly and reduces to zero at a critical field of $0.7 \mathrm{~V} / \AA ̊$, while it would open up again for larger electric field, suggesting a trivial topological phase transition. It is feasible to achieve the control of on/off functions about spin and charge in conducting channels by external electric field, which has substantial implications for promoting the development of QSH devices ${ }^{5,57-61}$, such as topological field-effect transistor (TFET). Here, we propose a conceptual design of TFET based on van der Waals heterostructures which are constructed by $\mathrm{AsBiI}_{2}$ monolayer and substrates with large band gap, such as $h$-BN, as shown in Fig. S7. In general, the van der Waals interaction has little influence on topological properties of monolayers, thus such multilayer structure can effectively increase the number of edge transport channels. The vertical electric field induced by the top and bottom gates can easily realize the on/off function. Additionally, we find that the topological phase of $\mathrm{SbBiI}_{2}$ monolayer is preserved under external electric field, manifesting an excellent robustness of QSH effect. The band gap shows a parabolic tendency with a maximum of $0.410 \mathrm{eV}$ at $0.3 \mathrm{~V} / \AA ̊$, which may be attributed to the competition between external electric field and internal polarized field. For $\mathrm{PAsI}_{2}, \mathrm{PSbI}_{2}, \mathrm{PBiI}_{2}$ and $\mathrm{AsSbI}_{2}$ monolayers, no topological transition can be observed under electric field, while their band gaps can be effectively tuned with a linear variation, as shown in Fig. S8. According to Bader analysis, we find the dipole moment of monolayers in vertical direction is along the positive orientation of $\mathrm{z}$ axis. When the negative electric field is applied, the dipole moment would be enhanced correspondingly due to the electron transfer from B to A atom, leading to the degeneracy further lifted. Thus, the energy levels near the Fermi 

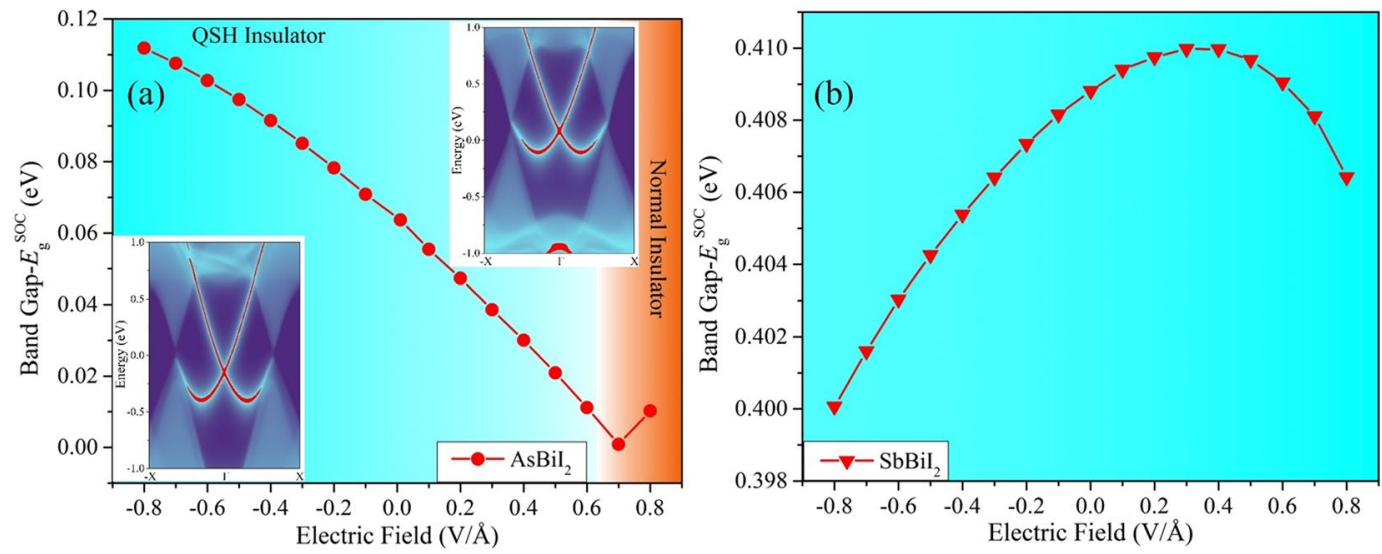

Figure 7. Variation of band gap $\left(E_{g}^{S O C}\right)$ as a function of external electric field for (a) $\mathrm{AsBiI}_{2}$ and (b) $\mathrm{SbBiI}_{2}$ monolayers, respectively. The left and right inserts of (a) are LDOSs of edges at $-0.5 \mathrm{~V} / \AA$ and $0.8 \mathrm{~V} / \AA \AA$, respectively.

level shift toward each other and the band gap decreases continuously. However, the positive electric field plays an opposite role. Such tunable band gap is highly favorable for applications in optoelectronic devices.

\section{Discussion}

Having established the existence of QSH phase in iodinated group-V binary monolayers, to further characterize the unconventional mechanism of band inversion, we investigate the variation of band gap at $\mathrm{K}$ point for all $\mathrm{ABI}_{2}$ monolayers as a function of SOC strength $\lambda / \lambda_{0}$, which is artificially set partial fractions of the original SOC strength $\lambda_{0}$, as shown in Fig. S9. The band gaps of $\mathrm{SbBiI}_{2}$ and $\mathrm{AsBiI}_{2}$ monolayers close at the relative SOC strength of 0.4 and 0.9 , respectively, and then reopen with larger SOC, indicating the occurrence of band inversion and topological phase. Similar result has been reported in Bi-III monolayers ${ }^{62}$, but it is distinctly different from the functionalized group-V films whose band gap would continuously increase with relative SOC strength. For $\mathrm{AsSbI}_{2}$ and $\mathrm{PBiI}_{2}$ monolayers, the band gaps reduce to zero when the $\lambda / \lambda_{0}$ are 1.2 and 1.7, respectively, suggesting that these two monolayers also have the feasibility to transform into QSH insulators by doping isoelectronic heavier elements with stronger SOC strength. However, until the relative SOC strength reaching 2.0, the band gaps closing and reopening cannot be observed for $\mathrm{PSbI}_{2}$ and $\mathrm{PAsI}_{2}$ monolayers due to their inherently weak SOC strength.

As compared with pristine $\mathrm{AB}$ monolayers, the intrinsic QSH effect in $\mathrm{ABI}_{2}$ monolayers is induced by I decoration. In fact, the states contributed by iodine atoms are far away from the Fermi level, and the role of iodination is orbital filtering effect (OFE), which has been reported in many kinds of $2 \mathrm{D}$ films $\mathrm{s}^{14,15,27-32,34,38}$. Considering that other halogen atoms $(\mathrm{F}, \mathrm{Cl}$, and $\mathrm{Br}$ ) and hydrogen atom are also feasible to achieve these effects, we wonder that: is the QSH effect relevant to decorated atom? If so, can the QSH effect still be characterized by such unconventional band inversion?

To verify it, we further investigate the electronic properties of hydrogenated $\mathrm{AB}$ monolayers $\left(\mathrm{ABH}_{2}\right)$. Their structural parameters are listed in Table S1 and illustrated in Fig. 1(e). One can see that the lattice constants and bond lengths of $\mathrm{ABH}_{2}$ monolayers are roughly equal to that in $\mathrm{ABI}_{2}$ monolayer, which also belong to space group P3M1. The optimized $h$ of $\mathrm{ABH}_{2}$ monolayers, however, becomes smaller clearly, except for $\mathrm{SbBiH}_{2}$, implying that the $\mathrm{A}-\mathrm{B}$ bond strength in $\mathrm{ABH}_{2}$ monolayers would be stronger than $\mathrm{ABI}_{2}$ monolayers. In the light of orbital-resolved band structures without SOC, as presented in Fig. S10, we can see that the orbital contributions in $\mathrm{ABH}_{2}$ monolayers are analogy to $\mathrm{ABI}_{2}$ monolayers, i.e., the states near the Fermi level are still determined by $\left|p_{\mathrm{x}, \mathrm{y}}^{-}\right\rangle$orbitals. Compared with $\mathrm{ABI}_{2}$ monolayers, with the exception of $\mathrm{SbBi}$, the band gaps of $\mathrm{ABH}_{2}$ are smaller, see Table S1, which is mainly attributed to the energy-level shift induced by bond strength. After considering SOC effect, the band gaps of $\mathrm{ABH}_{2}$ monolayers decrease due to band splitting, as shown in Fig. S11. Under this circumstance, the above-mentioned band inversion between $\left|p 2^{-}, \pm 3 / 2\right\rangle$ and $\left|p 1^{-}, \pm 1 / 2\right\rangle$ is only applicable to the $\mathrm{SbBiH}_{2}$ monolayer, which strongly points to the presence of QSH effect. For $\mathrm{AsBiH}_{2}$ monolayer, though the energy difference between $\left|p 2_{\mathrm{x}, \mathrm{y}}^{-}\right\rangle$and $\left|p 1_{\mathrm{x}, \mathrm{y}}^{-}\right\rangle$orbitals is much smaller, it is still irrelevant to band inversion. As a consequence, the emergence of topological phase in $\mathrm{AB}$ monolayers is also dependent on the functional atoms. Besides, the other hydrogenated $\mathrm{AB}$ monolayers also exhibit a trivial band order. The LDOS of edges for these $\mathrm{ABH}_{2}$ monolayers are further calculated and presented in Fig. S12. Indeed, only $\mathrm{SbBiH}_{2}$ monolayer shows the gapless helical edge states, spanning the bulk gap, which evidences the nontrivial topological nature. In a word, the intrinsic QSH effect of these $\mathrm{AB}$ monolayers is sensitive to the functional atoms, especially for $\mathrm{AsBi}$, and the unconventional band inversion can effectively characterize the nontrivial topological phase.

As is well known, the GGA exchange potential usually underestimates the band gap of semiconductors or insulators. To ensure the accuracy of band gap calculation, the more reliable hybrid HSE06 functional is adopted including the $\mathrm{SOC}$ effect. Here, the $\mathrm{AsBiI}_{2}$ and $\mathrm{AsBiH}_{2}$ monolayers are checked due to their smallest band gap in iodinated and hydrogenated systems, and the orbital projected band structures are presented in Fig. S13(a,b). We find that the band gap of $\mathrm{AsBiI}_{2}$ monolayer reduces to $0.019 \mathrm{eV}$. Combined with the atomic orbital contributions, 
this scenario can be understood by the fact that the band inversion vanishes with HSE06 correction, namely, a trivial topological phase formed. Even so, the nontrivial topology of $\mathrm{AsBiI}_{2}$ monolayer can be achieved easily with assistance of external factor, such as strain engineering, which still possesses the above excellent properties and potential application. However, for $\mathrm{AsBiH}_{2}$ monolayer, the band gap increases to $0.433 \mathrm{eV}$, maintaining its NI phase. Nevertheless, the calculated band structure of $\mathrm{SbBiH}_{2}$ with $\mathrm{HSE} 06$ functional is different from above cases, as shown in Fig. S13(c). Though its band gap is enhanced up to $0.545 \mathrm{eV}$, the band inversion between $\left|p_{\mathrm{x}, \mathrm{y}}^{-}\right\rangle$orbitals of $\mathrm{Sb}$ and $\mathrm{Bi}$ atoms is preserved, revealing that the band topology is robust.

\section{Conclusions}

In summary, we perform first-principles calculations to demonstrate the intrinsic QSH effect in $\mathrm{AsBiI}_{2}$ and $\mathrm{SbBiI}_{2}$ monolayers, which can be characterized by a unconventional band inversion of $\left|p_{\mathrm{x} y}^{-}\right\rangle$orbitals induced by SOC at $\mathrm{K}$ point. This nontrivial topological phase is verified by the explicit confirmation of $\mathrm{Z}_{2}$ invariant and gapless helical edge states, with a sizable bulk gap of $0.409 \mathrm{eV}$, which is sufficiently large for practical application at room temperature. Their topological properties can be effectively tuned by external factors, such as strain engineering and electric field, in which the electronically dominated transition between nontrivial and trivial phase supplies an avenue for manipulating the spin/charge conductance of edge state. These findings are of fundamental importance to theoretical and experimental studies of group- $\mathrm{V}$ binary monolayers, which provides an ideal platform to design optoelectronic and QSH devices.

\section{References}

1. Hasan, M. Z. \& Kane, C. L. Colloquium: Topological insulators. Rev. Mod. Phys. 82, 3045-3067 (2010).

2. Yan, B. \& Zhang, S. C. Topological materials. Rep. Prog. Phys. 75, 096501 (2012).

3. Qi, X.-L. \& Zhang, S.-C. Topological insulators and superconductors. Rev. Mod. Phys. 83, 1057-1110 (2011).

4. Žutić, I., Fabian, J. \& Das Sarma, S. Spintronics: Fundamentals and applications. Rev. Mod. Phys. 76, 323-410 (2004).

5. Pesin, D. \& MacDonald, A. H. Spintronics and pseudospintronics in graphene and topological insulators. Nat. Mater. 11, 409-416 (2012).

6. Garate, I. \& Franz, M. Inverse Spin-Galvanic Effect in the Interface between a Topological Insulator and a Ferromagnet. Phys. Rev. Lett. 104, 146802 (2010).

7. Bernevig, B. A., Hughes, T. L. \& Zhang, S.-C. Quantum Spin Hall Effect and Topological Phase Transition in HgTe Quantum Wells. Science 314, 1757-1761 (2006).

8. König, M. et al. Quantum Spin Hall Insulator State in HgTe Quantum Wells. Science 318, 766-770 (2007).

9. Zhang, H. et al. Topological insulators in Bi2Se3, Bi2Te3 and Sb2Te3 with a single Dirac cone on the surface. Nat. Phys. 5, 438-442 (2009).

10. Knez, I., Du, R.-R. \& Sullivan, G. Evidence for Helical Edge Modes in Inverted InAs/GaSb Quantum Wells. Phys. Rev. Lett. 107, 136603 (2011).

11. Kane, C. L. \& Mele, E. J. Quantum Spin Hall Effect in Graphene. Phys. Rev. Lett. 95, 226801 (2005).

12. Liu, C. C., Feng, W. \& Yao, Y. Quantum spin Hall effect in silicene and two-dimensional germanium. Phys. Rev. Lett. 107, 076802 (2011).

13. Liu, C.-C., Jiang, H. \& Yao, Y. Low-energy effective Hamiltonian involving spin-orbit coupling in silicene and two-dimensional germanium and tin. Phys. Rev. B 84, 195430 (2011).

14. Xu, Y. et al. Large-Gap Quantum Spin Hall Insulators in Tin Films. Phys. Rev. Lett. 111, 136804 (2013).

15. Zhao, H. et al. Unexpected Giant-Gap Quantum Spin Hall Insulator in Chemically Decorated Plumbene Monolayer. Sci. Rep. 6, 20152 (2016).

16. Yang, F. et al. Spatial and Energy Distribution of Topological Edge States in Single Bi(111) Bilayer. Phys. Rev. Lett. 109, 016801 (2012).

17. Zhang, P., Liu, Z., Duan, W., Liu, F. \& Wu, J. Topological and electronic transitions in a Sb(111) nanofilm: The interplay between quantum confinement and surface effect. Phys. Rev. B 85, 201410(R) (2012).

18. Kamal, C. \& Ezawa, M. Arsenene: Two-dimensional buckled and puckered honeycomb arsenic systems. Phys. Rev. B 91, 085423 (2015).

19. Drozdov, I. K. et al. One-dimensional topological edge states of bismuth bilayers. Nat. Phys. 10, 664-669 (2014).

20. Hirahara, T. et al. Interfacing 2D and 3D Topological Insulators: Bi(111) Bilayer on Bi2Te3. Phys. Rev. Lett. 107, 166801 (2011).

21. Wang, Y.-p et al. Tunable quantum spin Hall effect via strain in two-dimensional arsenene monolayer. J. Phys. D: Appl. Phys. 49, 055305 (2016).

22. Zhang, H., Ma, Y. \& Chen, Z. Quantum spin hall insulators in strain-modified arsenene. Nanoscale 7, 19152-9 (2015).

23. Zhao, M., Zhang, X. \& Li, L. Strain-driven band inversion and topological aspects in Antimonene. Sci. Rep. 5, 16108 (2015).

24. Zhang, H. \& Zhang, S.-C. Topological insulators from the perspective of first-principles calculations. Phys. Status Solid-R 7, 72-81 (2013).

25. Nie, Y., Rahman, M., Wang, D., Wang, C. \& Guo, G. Strain induced topological phase transitions in monolayer honeycomb structures of group-V binary compounds. Sci. Rep. 5, 17980 (2015).

26. Quensel, P., Ahlborg, K. \& Westgren, A. Minerals of the Varuträsk Pegmatite. Geologiska Föreningen i Stockholm Förhandlingar 59, 135-144 (1937)

27. Ji, W. X. et al. Stanene cyanide: a novel candidate of Quantum Spin Hall insulator at high temperature. Sci. Rep. 5, 18604 (2015).

28. Li, S. S. et al. Robust Room-Temperature Quantum Spin Hall Effect in Methyl-functionalized InBi honeycomb film. Sci. Rep. 6, $23242(2016)$

29. Li, S.-s, Ji, W.-x, Zhang, C.-w, Li, P. \& Wang, P.-j Robust room-temperature inversion-asymmetry topological transitions in functionalized HgSe monolayer. J. Mater. Chem. C 4, 2243 (2016).

30. Ma, Y. et al. Two-dimensional inversion-asymmetric topological insulators in functionalized III-Bi bilayers. Phys. Rev. B 91,235306 (2015).

31. Song, Z. et al. Quantum spin Hall insulators and quantum valley Hall insulators of $\mathrm{BiX} / \mathrm{SbX}(\mathrm{X}=\mathrm{H}, \mathrm{F}, \mathrm{Cl}$ and $\mathrm{Br})$ monolayers with a record bulk band gap. NPG Asia Mater. 6, e147 (2014).

32. Wang, Y.-p et al. Large-gap quantum spin Hall state in functionalized dumbbell stanene. Appl. Phys. Lett. 108, 073104 (2016).

33. Zhang, Q. \& Schwingenschlögl, U. Emergence of Dirac and quantum spin Hall states in fluorinated monolayer As and AsSb. Phys. Rev. B 93, 045312 (2016).

34. Zhang, R.-W. et al. Ethynyl-functionalized stanene film: a promising candidate as large-gap quantum spin Hall insulator. New J. Phys. 17, 083036 (2015).

35. Zhao, J., Li, Y. \& Ma, J. Quantum spin Hall insulators in functionalized arsenene (AsX, X=F, OH and $\mathrm{CH}$ ) monolayers with pronounced light absorption. Nanoscale 8, 9657-9666 (2016). 
36. Wang, Y. P. et al. Controllable band structure and topological phase transition in two-dimensional hydrogenated arsenene. Sci. Rep. 6, 20342 (2016)

37. Wang, D. et al. Robust large-gap quantum spin Hall insulators in chemically decorated arsenene films. New J. Phys. 18, 033026 (2016).

38. Niu, C. et al. Functionalized bismuth films: Giant gap quantum spin Hall and valley-polarized quantum anomalous Hall states. Phys. Rev. B 91, 041303(R) (2015).

39. Jin, K. H. \& Jhi, S. H. Quantum anomalous Hall and quantum spin-Hall phases in flattened Bi and Sb bilayers. Sci. Rep. 5, 8426 (2015).

40. Ji, W.-x et al. Giant gap quantum spin Hall effect and valley-polarized quantum anomalous Hall effect in cyanided bismuth bilayers. New J. Phys. 18, 083002 (2016).

41. Kresse, G. \& Hafner, J. Ab initio molecular-dynamics simulation of the liquid-metal-amorphous-semiconductor transition in germanium. Phys. Rev. B 49, 14251-14269 (1994).

42. Kresse, G. \& Furthmüller, J. Efficient iterative schemes for ab initio total-energy calculations using a plane-wave basis set. Phys. Rev. B 54, 11169-11186(1996).

43. Kresse, G. \& Joubert, D. From ultrasoft pseudopotentials to the projector augmented-wave method. Phys. Rev. B 59, 1758-1775 (1999).

44. Perdew, J. P. \& Wang, Y. Accurate and simple analytic representation of the electron-gas correlation energy. Phys. Rev. B 45, 13244-13249 (1992).

45. Grimme, S. Semiempirical GGA-type density functional constructed with a long-range dispersion correction. J. Comput. Chem. 27, 1787-1799 (2006).

46. Paier, J. et al. Screened hybrid density functionals applied to solids. J. Chem. Phys. 124, 154709 (2006).

47. Parlinski, K., Li, Z. Q. \& Kawazoe, Y. First-Principles Determination of the Soft Mode in Cubic ZrO2. Phys. Rev. Lett. 78, 4063-4066 (1997).

48. Mostofi, A. A. et al. wannier90: A tool for obtaining maximally-localised Wannier functions. Comput. Phys. Commun. 178, 685-699 (2008).

49. Sancho, M. P. L., Sancho, J. M. L. \& Rubio, J. Quick iterative scheme for the calculation of transfer matrices: application to Mo (100). J. Phys. F: Met. Phys. 14, 1205 (1984).

50. Sancho, M. P. L., Sancho, J. M. L., Sancho, J. M. L. \& Rubio, J. Highly convergent schemes for the calculation of bulk and surface Green functions. J. Phys. F: Met. Phys. 15, 851 (1985).

51. Zhang, R. W. et al. Functionalized Thallium Antimony Films as Excellent Candidates for Large-Gap Quantum Spin Hall Insulator. Sci. Rep. 6, 21351 (2016).

52. Ma, Y., Dai, Y., Kou, L., Frauenheim, T. \& Heine, T. Robust two-dimensional topological insulators in methyl-functionalized bismuth, antimony, and lead bilayer films. Nano Lett. 15, 1083-9 (2015).

53. Wittel, Ka \& M., R. Atomic spin-orbit interaction parameters from spectral data for 19 elements. Theor. Chim. Acta 33, 347-349 (1974).

54. Ezawa, M. A topological insulator and helical zero mode in silicene under an inhomogeneous electric field. New J. Phys. 14, 033003 (2012).

55. Soluyanov, A. A. \& Vanderbilt, D. Wannier representation ofZ2topological insulators. Phys. Rev. B 83, 035108 (2011).

56. Soluyanov, A. A. \& Vanderbilt, D. Computing topological invariants without inversion symmetry. Phys. Rev. B 83, 235401 (2011).

57. Brune, C. et al. Spin polarization of the quantum spin Hall edge states. Nat. Phys 8, 485-490 (2012).

58. Li, C. H. et al. Electrical detection of charge-current-induced spin polarization due to spin-momentum locking in Bi2Se3. Nat. Nano. 9, 218-224 (2014).

59. Mahfouzi, F., Nagaosa, N. \& Nikolić, B. K. Spin-Orbit Coupling Induced Spin-Transfer Torque and Current Polarization in Topological-Insulator/Ferromagnet Vertical Heterostructures. Phys. Rev. Lett. 109, 166602 (2012).

60. Mellnik, A. R. et al. Spin-transfer torque generated by a topological insulator. Nature 511, 449-451 (2014).

61. Wunderlich, J. et al. Spin Hall Effect Transistor. Science 330, 1801 (2010).

62. Chuang, F. C. et al. Prediction of large-gap two-dimensional topological insulators consisting of bilayers of group III elements with Bi. Nano Lett. 14, 2505-8 (2014).

\section{Acknowledgements}

This work was supported by the key program of NSFC (No. 11434006), the NBRP of China (Nos 2013CB922303 and 2015CB921502), 111 project (No. B13029), and the general program of NSFC (No. 11274143).

\section{Author Contributions}

S.L., C.W. and S.Y. conceived the study and wrote the manuscript. P.L. and S.H. performed the first-principles calculations. W.J calculated the phonon spectrum. T.Z. prepared figures. All authors read and approved the final manuscript.

\section{Additional Information}

Supplementary information accompanies this paper at doi:10.1038/s41598-017-05420-y

Competing Interests: The authors declare that they have no competing interests.

Publisher's note: Springer Nature remains neutral with regard to jurisdictional claims in published maps and institutional affiliations.

Open Access This article is licensed under a Creative Commons Attribution 4.0 International License, which permits use, sharing, adaptation, distribution and reproduction in any medium or format, as long as you give appropriate credit to the original author(s) and the source, provide a link to the Creative Commons license, and indicate if changes were made. The images or other third party material in this article are included in the article's Creative Commons license, unless indicated otherwise in a credit line to the material. If material is not included in the article's Creative Commons license and your intended use is not permitted by statutory regulation or exceeds the permitted use, you will need to obtain permission directly from the copyright holder. To view a copy of this license, visit http://creativecommons.org/licenses/by/4.0/.

(C) The Author(s) 2017 